\title{
4. Humanitäre Hilfe
}

\section{Gérard Perroulaz}

\section{OpenEdition \\ Journals}

Electronic version

URL: http://journals.openedition.org/sjep/110

DOI: $10.4000 /$ sjep. 110

ISSN: 1663-9677

\section{Publisher}

Institut de hautes études internationales et du développement

\section{Printed version}

Date of publication: 1 avril 2007

Number of pages: $43-56$

ISBN: 978-2-88247-067-6

ISSN: $1660-5926$

Electronic reference

Gérard Perroulaz, « 4. Humanitäre Hilfe », Schweizerisches Jahrbuch für Entwicklungspolitik [Online], 26-1 | 2007, Online erschienen am: 20 Mai 2010, abgerufen am 08 September 2020. URL : http:// journals.openedition.org/sjep/110 ; DOI : https://doi.org/10.4000/sjep.110 


\section{Humanitäre Hilfe*}

D ER BUNDESRAT hat im November 2006 seine neue „Botschaft über die Weiterführung der internationalen humanitären Hilfe der Eidgenossenschaft" vorgelegt. Die Botschaft vermittelt einen Überblick über die wichtigsten humanitären Aktionen der Direktion für Entwicklung und Zusammenarbeit (DEZA) seit 2001 und führt die aktuellen und künftigen Herausforderungen der humanitären Hilfe auf. Ein grosser Teil der Botschaft befasst sich ferner mit der Analyse der Beziehungen zu den hauptsächlichen Partnern der DEZA (staatliche und multilaterale Partner, Hilfswerke) und mit der Koordination mit den anderen im Ausland tätigen Bundesämtern (Aktionen zur Förderung von Frieden und Menschenrechten, Zusammenarbeit der Armee, Flüchtlingshilfe, Staatssekretariat für Wirtschaft). Der neue Finanzbetrag für die Jahre 2007 bis 2011 beläuft sich auf 1,5 Milliarden Franken, was dem Gesamtbetrag des vorhergehenden Rahmenkredits entspricht.

Die humanitäre Hilfe der Schweiz belief sich 2005 auf 303,7 Millionen Franken, 22,8 Millionen mehr als im Vorjahr. Die Haupteinsatzgebiete waren (in absteigender Reihenfolge der ausgezahlten Beträge) die vom Tsunami im Dezember 2004 betroffenen Länder in Asien, der Sudan (Darfurkrise), Afghanistan, die Region der Grossen Seen in Afrika, Pakistan (nach dem Erdbeben), der Kaukasus und Palästina. Rund ein Drittel der humanitären Hilfe der Schweiz entfällt auf das Internationale Komitee vom Roten Kreuz (IKRK). Die direkten Einsätze des Schweizerischen Korps für humanitäre Hilfe (SKH) machen ein Fünftel der humanitären Hilfe aus. 37 Prozent der humanitären Hilfe läuft über UN-Organisationen, vor allem über das Welternährungsprogramm (WFP), das Hochkommissariat für Flüchtlinge (UNHCR) und das Hilfswerk für Palästinaflüchtlinge im Nahen Osten (UNRWA). 8 Prozent der öffentlichen humanitären Hilfe wird über die Partnerhilfswerke der DEZA geleitet.

\subsection{Neuer Rahmenkredit für die humanitäre Hilfe 2007-2011}

Am 29. November 2006 hat der Bundesrat die Botschaft über die Weiterführung der internationalen humanitären Hilfe der Eidgenossenschaft ${ }^{1}$ verabschiedet. Der Rahmenkredit setzt den Gesamtbetrag für die Finanzierung der Einsätze humanitärer Hilfe auf 1,5 Milliarden Franken für die Laufzeit von Mitte 2007 bis 2011 fest. Im ersten Teil der Botschaft werden die gegenwärtigen humanitären Herausforderungen sowie die Stossrichtungen der humanitären Hilfe für die nächsten Jahre angeführt. Im zweiten Teil wird zu den von 2001 bis 2005 erfolgten Einsätzen Bilanz gezogen. Ferner werden die Beziehungen der humanitären Hilfe zu den übrigen Bereichen der DEZA und den anderen Ämtern der Bundesverwaltung beschrieben.

* Von Gérard Perroulaz, Lehr- und Forschungsbeauftragter am IUED.

1 Bundesrat, Botschaft über die Weiterführung der internationalen humanitären Hilfe der Eidgenossenschaft, vom 29. November 2006, (BB1 2006, 9617). 


\subsubsection{Herausforderungen der humanitären Hilfe}

Die humanitäre Hilfe muss sich zahlreichen Herausforderungen stellen, welche komplex und oft nicht sofort lösbar sind. Die Zahl der zwischenstaatlichen Konflikte wie auch die Anzahl der Flüchtlinge ist zwar seit Anfang der 90er Jahre beträchtlich zurückgegangen, hingegen weist die Botschaft auf die starke Zunahme der in den Ländern intern Vertriebenen hin, deren Anzahl zwischen 1982 und 2003 von 3 auf 23 Millionen gestiegen ist. Die Art der Konflikte hat sich somit verändert. An die Stelle des klassischen Kriegs zwischen zwei Staaten ist eine Vielzahl interner Konflikte zwischen zahlreichen bewaffneten Gruppen und mit erhöhter Gewalt gegen die Zivilbevölkerung getreten. Der Zugang zu den Opfern und die Einhaltung des humanitären Völkerrechts sind schwieriger geworden. Jede Region hat ihre eigenen Probleme in einem besonderen historischen, kulturellen und sozialen Umfeld. Die Regierungsfähigkeit der Staaten ist angesichts der Kriminalisierung von Konflikten - namentlich in Form von Menschenhandel oder Handel mit Edelmetallen und Energieträgern zum Kauf von Waffen - oftmals geschwächt.

Gewisse humanitäre Krisensituationen sind oft stark mediatisiert, was positive wie auch negative Aspekte hat. Die Mediatisierung ermöglicht es, Aufmerksamkeit zu erregen, finanzielle Ressourcen zu mobilisieren und zu konzentrieren, um der betroffenen Bevölkerung zu Hilfe zu kommen. Jedoch können diese Ressourcen dann für andere, weniger mediatisierte Krisen fehlen. Eine Stärkung der Einsatzkapazitäten der internationalen humanitären Hilfe ist notwendig.

Weiter hebt die Botschaft des Bundesrates hervor: „Humanitäre Krisen könnten oft vermieden werden. Hinter einer Dürre, einem Konflikt oder einer technologischen Katastrophe verbirgt sich meistens eine Vielfalt von Faktoren: Armut, soziale Ungerechtigkeit, schlechte Regierungsführung, Klimawandel und das Fehlen von Strategien zur Vorbereitung auf solche Risiken“"2.

\subsubsection{Aufgabenfelder der internationalen humanitären Hilfe des Bundes}

Die DEZA hat die Schwerpunkte der internationalen humanitären Hilfe der Eidgenossenschaft (nachstehend „Humanitäre Hilfe des Bundes“) in ihrer Strategie 2005 der humanitären $\mathrm{Hilfe}^{3}$ festgelegt. Der Bund entfaltet seine humanitäre Tätigkeit auf bilateraler und multilateraler Ebene in den vier folgenden Aufgabenbereichen:

๑ Prävention mit dem Ziel, die Opferzahl und die Schäden bei Naturkatastrophen zu reduzieren. Die DEZA hat beschlossen, der Schaffung von Synergien zwischen der humanitären Hilfe und den Programmen der Entwicklungszusammenarbeit im Präventionsbereich grössere Aufmerksamkeit zukommen zu lassen.

- Nothilfe zur Rettung und Überlebenssicherung von Menschen, die von Katastrophen und Konflikten heimgesucht werden. Die direkte Nothilfe des Schweizerischen Korps für humanitäre Hilfe (SKH) verfügt über mehrere

2 Botschaft über die Weiterführung der internationalen humanitären Hilfe der Eidgenossenschaft, op. cit., S. 9625.

3 DEZA, Solidarität leben. Humanitäre Hilfe, Strategie 2005. Bern, DEZA, 2002, <http://www.deza. admin.ch>. 
Interventionsmechanismen, darunter Soforteinsatzteams, um die Bedürfnisse zu analysieren, erste Nothilfemassnahmen zu ergreifen und die Koordination mit den anderen Akteuren der internationalen Hilfe vor Ort sicherzustellen. Die Rettungskette Schweiz kommt bei Erdbeben zum Einsatz, um verschüttete Personen aus den Trümmern zu bergen und erste Hilfe zu leisten. Die Überlebenshilfe ermöglicht anschliessend, Notunterkünfte zu errichten und die Versorgung mit Trinkwasser und Nahrungsmitteln zu gewährleisten.

- Wiederaufbau nach Katastrophen und Konflikten, um die Basisinfrastruktur (Krankenhäuser, Schulen, Unterkünfte, Verkehrsverbindungen, usw.) wieder instand zu setzen. Ferner geht es darum, Güter und Material zur Wiederaufnahme der landwirtschaftlichen Tätigkeit oder für die Reparatur von Häusern bereitzustellen und die Rückkehr der Flüchtlinge zu ermöglichen.

- Schutz- und Anwaltschaft für die Opfer und deren Rechte. Die DEZA unterstützt humanitäre Akteure, die ein spezifisches Schutzmandat haben. Sie kann auch die Aufmerksamkeit der Behörden auf betroffenene Personen lenken, die Öffentlichkeit sensibilisieren und an die Verpflichtung erinnern, das humanitäre Völkerrecht zu respektieren und für dessen Einhaltung zu sorgen. Diese Aktionen sollen in Zukunft noch verstärkt werden.

Die DEZA wacht bei ihrer Tätigkeit über die Anwendung des Prinzips Do No Harm. Das Prinzip erleichtert die systematische Festlegung von Methoden zur Umsetzung der humanitären Hilfe und der Entwicklungszusammenarbeit, die bei der Regelung von Konflikten zur Stärkung der lokalen Kräfte für die Förderung von Frieden und Entwicklung beitragen sollen, anstatt Spannungen mangels Aufmerksamkeit zu schüren.

\subsubsection{Beziehungen der DEZA zu anderen Bundesämtern}

Die DEZA setzt die Politik der humanitären Hilfe der Schweiz um. Diese Politik hat jedoch offensichtliche Zusammenhänge mit anderen Tätigkeitsbereichen des Bundes: humanitäres Völkerrecht, Beteiligung an der Definition der Politik und der Praktiken auf internationaler Ebene (Policy-making), Menschenrechtspolitik, Politik der Flüchtlingsaufnahme, Friedensförderung, längerfristige Entwicklungszusammenarbeit. Die Botschaft setzt den Schwerpunkt besonders auf die erforderliche Abstimmung und Koordination mit anderen Tätigkeiten der Bundesverwaltung, wobei sie folgende Aspekte herausstellt:

- Entwicklungspolitik. Friede und Stabilität werden auch durch langfristige Aktionen zur Armutsbekämpfung und zur Förderung von Menschenrechten, Demokratie und Rechtsstaatlichkeit gesichert. Da die Nothilfe sich nicht von den langfristigen Aktionen trennen lässt, muss der Konfliktverhütung im Rahmen von Programmen der Entwicklungszusammenarbeit und einer gleichzeitigen Umsetzung der Wiederaufbaumassnahmen und der Massnahmen langfristiger Zusammenarbeit nach Konflikten besondere Aufmerksamkeit zukommen.

- Friedenspolitik. Die Einsätze der humanitären Hilfe werden durch Massnahmen der zivilen Friedensförderung und der Stärkung der Menschenrechte ergänzt, die von der Politischen Abteilung IV „Menschliche Sicherheit“ durchgeführt werden. Die Förderung von Frieden, Rechtsstaatlichkeit und 
Menschenrechten kann dazu beitragen, gewisse Krisen zu verhüten und die Schwierigkeiten beim Zugang zu Opfern von Krisen und Konflikten zu lösen.

- Vertreibung und Migration. Die Präventionsmassnahmen und die Nothilfe tragen dazu bei, Zwangsvertreibungen der betroffenen Bevölkerung zu begrenzen und die Vertreibung auf nahe gelegene Gebiete zu beschränken. Die Unterstützung beim Wiederaufbau ermöglicht die raschere Rückkehr der Flüchtlinge in ihre Ursprungsregion. In der Schweiz wird die Interdepartementale Leitungsgruppe Rückkehrhilfe (ILR) vom Bundesamt für Migration (BFM) und der DEZA gemeinsam geführt. Dieses Organ plant die Programme zur Hilfe für die Rückkehr von Flüchtlingen, die dann vom Bundesamt für Migration finanziert und unter der Leitung der DEZA umgesetzt werden.

- Wirtschaftssanktionen. Für die Schweiz ist darauf zu achten, dass die internationalen Sanktionen keine negativen Auswirkungen auf die Zivilbevölkerung oder die Wirtschaft anderer Länder nach sich ziehen.

- Zivil-militärische Beziehungen. Dieser Bereich hat in den letzten Jahren international Bedeutung erlangt und wirft wichtige Fragen auf ${ }^{4}$. Die Schweizer Armee leistet verschiedene Formen der Unterstützung in Krisengebieten: Entsendung von Hubschraubern zur Beförderung der Nahrungsmittelhilfe und Nothilfe. Entsendung von Material (z.B. Notspitalausrüstung), Finanzierung von Entminungsaktionen. Gemäss dem Bundesrat müssen die Beziehungen zwischen den zivilen und militärischen Einsatzkräften stets unter Berücksichtigung der international anerkannten humanitären Grundsätze gestaltet werden. Eine militärische Unterstützung muss einen subsidiären Charakter haben - sie ergänzt die zivilen Mittel - und der zivilen Förderung unterstellt werden ${ }^{5}$. Der zivile und der militärische Teil der Friedensmission müssen sich ergänzen. Die humanitären Aktionen müssen von den Sicherheitsoperationen klar getrennt werden und dürfen nicht der Verfolgung von Sicherheitszielen dienen.

\subsubsection{Einsatz der Mittel für die Periode 2001-2005}

Die Botschaft des Bundesrates erstattet Bericht über die von 2001 bis 2005 umgesetzten Aktionen. Sie führt konkrete Beispiele an für dank der Humanitären Hilfe des Bundes durchgeführte Einsätze, wie Hilfe an Vertriebene in Darfur, Hilfe ans südliche Afrika (schwierige Situation infolge der dreifachen Bedrohung durch die chronische Ernährungskrise, die HIV-Aids-Pandemie und leistungsschwache Regierungen), Schutz und Anwaltschaft für die Opfer von als Kriegswaffe eingesetzter Vergewaltigung in der Region der grossen Seen in Afrika, Hilfe für Osteuropa nach den Überschwemmungen im Sommer 2002, fortlaufende Unterstützung Weissrusslands nach der Reaktorkatastrophe in Tschernobyl, Hilfe für die Flüchtlinge im Nordkaukasus (Südossetien und Tschetschenien), Verhütung von Naturkatastrophen in den Andenstaaten.

4 Siehe insbesondere die Analyse der Organisation Caritas bezüglich der humanitären Herausforderungen und der zivil-militärischen Zusammenarbeit: Caritas, Les défis humanitaires. Les dilemmes politiques de l'aide d'urgence, coll. Prise de position, Nr. 11, Luxemburg, Caritas Luxemburg; Luzern, Caritas Schweiz, November 2005, 164 S. Der Bericht stellt die politischen Dilemmata der Politik der humanitären Hilfe sowie die Risiken bezüglich Nebenwirkungen und Instrumentalisierung heraus.

5 Botschaft über die Weiterführung der internationalen humanitären Hilfe der Eidgenossenschaft, op. cit., S. 9642. 
Die Grafik 4.1 gibt zeigt die geografische Aufteilung der humanitären Hilfe für die Jahre 2001-2005. Afrika war das grösste Einsatzgebiet der humanitären Hilfe (mit 22,1\% des Gesamtbetrags), gefolgt von Osteuropa und der GUS $(14,4 \%)$, Asien (12,8\%), dem Mittleren Osten (7,8\%) und Lateinamerika $(5,8 \%)$. Der relative hohe Anteil der geografisch nicht aufgeteilten Mittel erklärt sich insbesondere durch die Beiträge an die internationalen Organisationen, einschliesslich des Internationalen Komitees vom Roten Kreuz (IKRK).

Der kumulierte Gesamtbetrag der humanitären Hilfe für die Jahre 2001-2005 belief sich auf 1410,9 Millionen Franken. Davon wurden 64 Prozent (902 Millionen Franken) an die internationalen Organisationen und das IKRK ausgezahlt (siehe Grafik 4.2). 10 Prozent (143,3 Millionen Franken) dienten zur Finanzierung humanitärer Einsätze von Nichtregierungsorganisationen (NRO). Die direkten Aktionen des Schweizerischen Korps für humanitäre Hilfe (SKH) machten 19 Prozent der Hilfe (272 Millionen Franken) aus.

Grafik 4.1: Geografische Aufteilung der humanitären Hilfe der Schweiz, 2001-2005

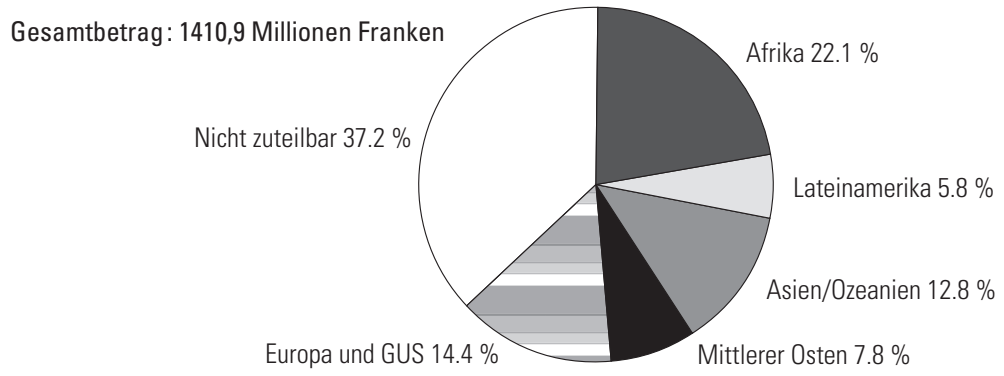

\section{Grafik 4.2: Aufteilung der humanitären Hilfe der Schweiz nach internationalen Organisationen, 2001-2005}

Gesamtbetrag :

902,1 Millionen Franken

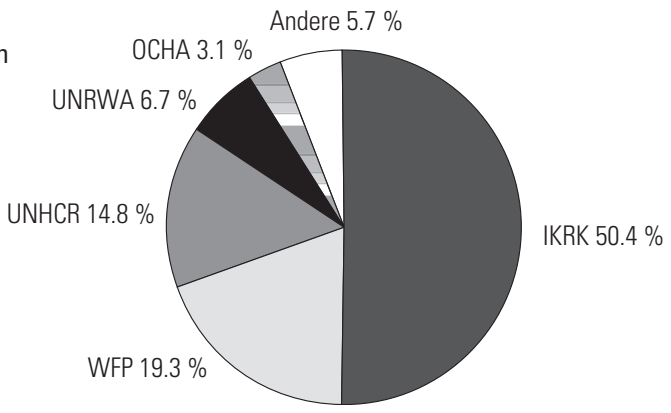

Quelle der Grafiken 4.1 und 4.2: Bundesrat, Botschaft über die Weiterführung der internationalen humanitären Hilfe der Eidgenossenschaft, vom 29. November 2006, (BBI 2006, 9617). S. 9670-9671.

Neben dem IKRK unterhält die Schweiz vor allem Beziehungen zu fünf multilateralen Partnern, nämlich zum Welternährungsprogramm (WFP), zum UNHochkommissariat für Flüchtlinge (UNHCR), zum UN-Hilfswerk für Palästina- 
flüchtlinge im Nahen Osten (UNRWA), zum UN-Büro für die Koordination humanitärer Angelegenheiten (OCHA) und zu der Internationalen Strategie zur Reduzierung von Katastrophen (ISDR). Andere internationale Organisationen sind ebenfalls Partner für die Humanitäre Hilfe des Bundes, namentlich das Büro für Krisenvorbeugung und Wiederaufbau des UN-Entwicklungsprogramms (UNDP/BCPR), die Internationale Föderation der Rotkreuz- und Rothalbmondgesellschaften (IFRCRCS), der Kinderhilfsfonds der Vereinten Nationen (UNICEF) und die Internationale Organisation für Migration (IOM).

\subsubsection{Zuteilung der Mittel des neuen Rahmenkredits der humanitären Hilfe}

Die Aufteilung des für eine Laufzeit von mindestens vier Jahren vorgesehenen Finanzbetrags ist aus Tabelle 4.1 ersichtlich. Gegenüber dem vorherigen Rahmenkredit sieht die DEZA vor, die Nahrungsmittelhilfe und in geringerem Masse die Finanzhilfe zugunsten einer Erhöhung der Mittel für die direkten Einsätze und das IKRK zu reduzieren. Der Gesamtbudgetbetrag liegt jedoch unverändert bei 1,5 Milliarden Franken. Da der vorhergehende Rahmenkredit gegen Mitte des Jahres 2007 ausgeschöpft sein wird, muss das Parlament die neue Botschaft in seiner Sommersession 2007 prüfen.

\section{Tabelle 4.1: Aufteilung der Mittel gemäss den letzten drei Rahmenkrediten der humanitären Hilfe (in Millionen Franken)}

\begin{tabular}{lccc}
\hline Mittel der humanitären Hilfe & $\begin{array}{c}\text { Rahmenkredit } \\
\mathbf{1 9 9 8 - 2 0 0 1}\end{array}$ & $\begin{array}{c}\text { Rahmenkredit } \\
\mathbf{2 0 0 2 - 2 0 0 6}\end{array}$ & $\begin{array}{c}\text { Rahmenkredit } \\
\mathbf{2 0 0 7 - 2 0 1 1}\end{array}$ \\
\hline Direkte Einsätze mit Angehörigen des SKH & 110 & 180 & 190 \\
\hline Beiträge an das Sitzbudget und an die Feldaktionen des IKRKa & 91 & 420 & 460 \\
\hline Finanzielle Unterstützung an humanitäre Aktionen & 489 & 550 & 540 \\
\hline Nahrungsmittelhilfe mit schweizerischen Milchprodukten & 140 & 140 & 120 \\
\hline Nahrungsmittelhilfe mit Getreide & 110 & 100 & 80 \\
\hline $\begin{array}{l}\text { Verpflichtungsreserve für Katastrophen und Notlagen, } \\
\text { die nicht durch die Zahlungskredite gedeckt werden können }\end{array}$ & 110 & 110 & 110 \\
\hline Gesamtbetrag & $\mathbf{1 0 5 0}$ & $\mathbf{1 5 0 0}$ & $\mathbf{1 5 0 0}$ \\
\hline
\end{tabular}

Quellen: Bundesrat, Botschaft über die Weiterführung der internationalen humanitären Hilfe der Eidgenossenschaft, vom 29. November 2006, (BBI 2006, 9617). S. 9654.

Bundesrat, Botschaft über die Weiterführung der internationalen humanitären Hilfe der Eidgenossenschaft, vom 14. November 2001, (BBI 2002, 2221).

a Die Beiträge an das IKRK enthalten den Beitrag an das Sitzbudget und die Auszahlungen für die Aktionen im Feld. Bis 1997 wurde der Beitrag an das Sitzbudget des IKRK durch einen getrennten Rahmenkredit zugeteilt.

b Finanzierung der von schweizerischen oder internationalen Partnerorganisationen durchgeführten Aktionen.

c Die Verpflichtungsreserve gestattet es dem Bundesrat, auf Krisensituationen zu reagieren, ohne die Jahresbudgetstruktur der Humanitären Hilfe des Bundes ändern zu müssen.

\subsection{Eckdaten der Humanitären Hilfe des Bundes für 2005}

4.2.1. Überblick über die Humanitäre Hilfe der Schweiz und ihrer wichtigsten Partner im Jahr 2005

Die humanitäre Hilfe der DEZA belief sich 2005 auf 303,7 Millionen Franken (gegenüber 280,9 Millionen im Jahr 2004). Davon entfielen 296,8 Millionen Franken auf die Entwicklungsländer und der Rest auf die Oststaaten, die nicht in der öffentlichen Hilfe für Entwicklungsländer und -gebiete inbegriffen sind. 
Tabelle 4.2 gibt einen Überblick über die im Jahr 2005 ausgezahlte bilaterale und multilaterale humanitäre Hilfe und über die wichtigsten Partner der DEZA ${ }^{6}$. 113 Millionen Franken an humanitärer Hilfe liefen über die internationalen Organisationen und 94,5 Millionen Franken wurden zusätzlich über die Organisationen des Internationalen Roten Kreuzes geleitet ${ }^{7}$. Insgesamt wurden im Jahr 200568 Prozent der humanitären Hilfe über internationale Organisationen ausgezahlt, jedoch sind lediglich die allgemeinen Beiträge an die internationalen Organisationen (25,6 Millionen Franken, d.h. 8,4\% der gesamten Hilfe) in der multilateralen Hilfe inbegriffen.

Tabelle 4.2: Humanitäre Hilfe der DEZAa für 2005 (in Millionen Franken)

\begin{tabular}{|c|c|c|c|c|}
\hline & $\begin{array}{c}\text { Allgemeine } \\
\text { Beiträge }\end{array}$ & $\begin{array}{c}\text { Spezifische } \\
\text { Beiträge }\end{array}$ & $\begin{array}{l}\text { Nahrungs- } \\
\text { mittelhilfe }\end{array}$ & $\begin{array}{c}\text { Gesamt- } \\
\text { betrag }\end{array}$ \\
\hline Internationale Organisationen & ${ }^{\mathrm{b}} 25.6$ & 68.0 & 19.3 & 113.0 \\
\hline Welternährungsprogramm (WFP) & 1.5 & 24.1 & 17.7 & 43.4 \\
\hline UN-Hochkommissariat für Flüchtlinge (UNHCR) & 11.0 & 16.8 & & 27.8 \\
\hline $\begin{array}{l}\text { UN-Hilfswerk für Palästinaflüchtlinge im } \\
\text { Nahen Osten (UNRWA) }\end{array}$ & 10.5 & 0.8 & & 11.3 \\
\hline $\begin{array}{l}\text { UN-Büro für die Koordination humanitärer } \\
\text { Angelegenheiten (UNOCHA) }\end{array}$ & 1.8 & 5.9 & & 7.6 \\
\hline Kinderhilfsfonds der UNO (UNICEF) & & 4.3 & & 4.3 \\
\hline Andere internationale Organisationen & 0.9 & 16.1 & 1.6 & 18.6 \\
\hline Organisationen des Internationalen Roten Kreuzes & 71.0 & 23.5 & & 94.5 \\
\hline Internationales Komitee vom Roten Kreuz (IKRK) & 70.0 & 22.9 & & 92.9 \\
\hline $\begin{array}{l}\text { Internationale Föderation der Rotkreuz- und } \\
\text { Rothalbmondgesellschaften (IFRCRCS) }\end{array}$ & 1.0 & 0.6 & & 1.6 \\
\hline Schweizerische NRO & & 14.3 & 10.8 & 25.1 \\
\hline $\begin{array}{l}\text { Direkte Aktionen des Schweizerischen Korps } \\
\text { für humanitäre Hilfe (SKH) }\end{array}$ & & 56.7 & 0.5 & 57.2 \\
\hline Andere Aktionen der humanitären Hilfe & & 11.5 & 2.4 & 13.9 \\
\hline Gesamtbetrag der humanitären Hilfe 2005 & 96.6 & 174.0 & 33.0 & 303.7 \\
\hline
\end{tabular}

Quelle: DEZA/SECO, Jahresbericht der internationalen Zusammenarbeit der Schweiz 2005, Bern, 2006, S. 18.

a umfasst die bilaterale und die multilaterale humanitäre Hilfe.

b Die multilaterale Hilfe der DEZA besteht nur aus den allgemeinen Beiträgen an die humanitären UN-Organisationen (gemäss DACLeitlinien). Die bilaterale humanitäre Hilfe umfasst alle anderen Zahlungen: direkte Aktionen des SKH, Beiträge an NRO, Zahlungen an das IKRK, spezifische Beiträge an die internationalen Organisationen sowie die gesamte Nahrungsmittelhilfe (einschliesslich der Hilfe über das Welternährungsprogramm).

Die Nichtregierungsorganisationen erhielten von der DEZA 25,1 Millionen Franken zur Finanzierung ihrer humanitären Aktionen. Der Beitrag des Bundes zur Finanzierung von Projekten der NRO darf grundsätzlich 50 Prozent ihrer Kosten nicht übersteigen. Die wichtigsten NRO, denen diese Beträge 2005 zugute kamen, waren Caritas Schweiz (5,4 Millionen Franken), Terre des Hommes (3,9 Millionen Franken) und das Schweizerische Rote Kreuz (2,8 Millionen Franken). Die direkten Aktionen des Schweizerischen Korps für humanitäre Hilfe (SKH) beliefen sich 2005 auf 57,2 Millionen Franken (gegenüber 38,4 Millionen im Vorjahr). Die Anzahl der Einsätze des SKH hat sich mit insgesamt 389 Interventionen im Jahr 2005 deutlich erhöht, vor allem in Asien (mit 228 Interventionen

6 Zusätzliche Informationen über die Partner der DEZA finden sich unter <http://www.deza.admin.ch> $>$ Aktivitäten $>$ Humanitäre Hilfe $>$ Partnerorganisationen.

7 Das IKRK wird vom Entwicklungshilfeausschuss der OECD (DAC) als internationale Nichtregierungsorganisation angesehen, somit sind die Beiträge an das IKRK in der bilateralen öffentlichen Hilfe enthalten. 
gegenüber 98 im Vorjahr). Das SKH ist ein Milizkorps und umfasst 700 Personen, die für den Katastropheneinsatz bereit sind und nach Fachgruppen (Bau, Logistik, Information, Medizin, Rettung, Trink- und Abwasser) rekrutiert werden. Die Einsatztage im Jahr 2005 entsprachen 96 Vollzeitstellen.

Die Nahrungsmittelhilfe erreichte 200533 Millionen Franken. Davon liefen 19,3 Millionen über die internationalen Organisationen und 10,8 Millionen über die NRO. Die Nahrungsmittelhilfe umfasst schweizerische Milchprodukte (2005 : 19 Millionen Franken an gebundener Hilfe) sowie Getreide (14 Millionen Franken), das meist vor Ort oder in der näheren Umgebung gekauft wird.

Seit 2005 hat der Bund das Programm für die Weiterverwendung von Armeematerial (WAM) eingerichtet. Das Programm hat die Bereitstellung von überschüssigem Armeematerial zum Ziel, welches für Projekte der DEZA und anderer Organisationen mit ähnlichem Profil wiederverwendet werden kann. 2005 hat die Schweiz erstmals die Lieferung von Militärmaterial für die Humanitäre Hilfe in die Berechnung der öffentlichen Entwicklungshilfe eingeschlossen. Im Zusammenhang mit der Reorganisation der Schweizer Armee stösst die Armee Material ab, das im Rahmen von Entwicklungsprojekten oder humanitären Einsätzen weiterverwendet werden kann (medizinisches Material, Fahrzeuge, Werkzeuge, Textilien, Spitaleinheiten $)^{8}$. So wurden beispielsweise im Oktober 2005 von der Armee stammende sanitäre Container an Pakistan in ein vom Erdbeben betroffenes Gebiet geliefert.

\subsubsection{Geografische Aufteilung der humanitären Hilfe im Jahr 2005}

Die Haupteinsatzgebiete der bilateralen humanitären Hilfe der DEZA im Jahr 2005 waren der Sudan, das Gebiet der Grossen Seen, der Kaukasus, Palästina, das vom Tsunami betroffene Katastrophengebiet in Asien, Pakistan und Afghanistan (siehe Tabelle 4.3).

Nach der Tsunamikatastrophe ist der Anteil der bilateralen humanitären Hilfe für die asiatischen Länder merklich gestiegen und machte 2005 26,5 Prozent der Hilfe (gegenüber 18,3\% im Vorjahr) aus. Nach Beendigung der Konflikte in Südosteuropa ging der Anteil Europas zwischen 2002 und 2005 stark zurück und fiel von 24 auf 6 Prozent der humanitären Hilfe ab.

Tabelle 4.3: Bilaterale humanitäre Hilfe der DEZA für 2005 und die drei vorhergehenden Jahre (in Millionen Franken)

\begin{tabular}{crrrr}
\hline & $\mathbf{2 0 0 2}$ & $\mathbf{2 0 0 3}$ & $\mathbf{2 0 0 4}$ & $\mathbf{2 0 0 5}$ \\
\hline Afrika & 59.1 & 61.0 & 70.9 & 65.6 \\
\hline davon Sudan & 5.7 & 6.9 & 13.6 & 13.1 \\
\hline Kongo (DR) & 5.6 & 5.6 & 4.1 & 5.7 \\
\hline Angola & 8.2 & 7.8 & 7.6 & 5.3 \\
\hline Burundi & 3.7 & 3.0 & 4.6 & 4.6 \\
\hline Liberia & 2.6 & 3.7 & 4.0 & 3.8 \\
\hline Uganda & 0.1 & 1.0 & 3.4 & 3.7 \\
\hline Tschad & 0.3 & 0.7 & 3.9 & 3.1 \\
\hline Sierra Leone & 4.5 & 3.5 & 3.6 & 2.1 \\
\hline Elfenbeinküste & 1.7 & 1.5 & 1.1 & 2.0 \\
\hline
\end{tabular}

8 Siehe Projekt WAM unter <http://www.deza.admin.ch $>>$ Projekte $>$ Weiterverwendung Armee Material. 


\begin{tabular}{|c|c|c|c|c|c|}
\hline & & 2002 & 2003 & 2004 & 2005 \\
\hline \multicolumn{2}{|l|}{ Asien } & 49.3 & 49.3 & 45.8 & 73.8 \\
\hline \multicolumn{2}{|c|}{ davon Indonesien } & 2.1 & 1.3 & 1.8 & 12.3 \\
\hline \multicolumn{2}{|c|}{ Afghanistan } & 16.9 & 14.5 & 12.0 & 11.5 \\
\hline \multicolumn{2}{|c|}{ Sri Lanka } & 3.2 & 2.8 & 3.0 & 8.8 \\
\hline \multicolumn{2}{|c|}{ Pakistan } & 0.2 & 1.2 & 2.6 & 7.9 \\
\hline \multicolumn{6}{|c|}{ Gebiet unter palästinensischer } \\
\hline & Verwaltung & 2.1 & 5.5 & 5.8 & 7.0 \\
\hline & Georgien & 3.8 & 6.1 & 5.3 & 5.2 \\
\hline & Thailand & 1.3 & 1.4 & 1.4 & 4.2 \\
\hline \multicolumn{2}{|c|}{ Europa } & 64.2 & 23.4 & 19.1 & 17.4 \\
\hline \multirow[t]{4}{*}{ davon } & Russland & 14.4 & 6.2 & 5.5 & 5.8 \\
\hline & Weissrussland & 2.6 & 2.3 & 3.1 & 3.2 \\
\hline & Moldawien & 3.1 & 4.2 & 3.3 & 3.1 \\
\hline & Serbien-Montenegro & 8.5 & 5.6 & 3.1 & 1.5 \\
\hline \multicolumn{2}{|c|}{ Lateinamerika } & 14.0 & 13.5 & 16.0 & 22.0 \\
\hline \multirow[t]{4}{*}{ davon } & Kolumbien & 4.3 & 5.0 & 4.8 & 5.2 \\
\hline & Kuba & 0.7 & 0.7 & 1.0 & 4.8 \\
\hline & Haiti & 0.1 & 0.1 & 2.8 & 3.2 \\
\hline & Peru & 3.2 & 2.0 & 3.1 & 2.6 \\
\hline \multicolumn{2}{|c|}{ Geografisch nicht aufgeschlüsselta } & 99.6 & 97.0 & 98.2 & 99.2 \\
\hline \multicolumn{2}{|c|}{ Bilaterale Hilfe insgesamt $^{b}$} & 270.3 & 244.2 & 250.0 & 277.8 \\
\hline
\end{tabular}

Quellen: Statistischer Dienst der DEZA. DEZA/SECO, Jahresbericht der internationalen Zusammenarbeit der Schweiz 2005, Ausgaben 2003 bis 2005.

a umfasst die Beiträge an das Internationale Komitee vom Roten Kreuz (IKRK) und an die Internationale Föderation der Rotkreuzund Rothalbmondgesellschaften (IFRCRCS).

b Gesamtbetrag der bilateralen humanitären Hilfe der DEZA für die Entwicklungsländer und Transitionsländer. Die bilaterale humanitäre Hilfe umfasst die direkten Aktionen des SKH, die Beiträge an die NRO, die Zahlungen an das IKRK, die spezifischen Beiträge an die internationalen Organisationen sowie die gesamte Nahrungsmittelhilfe (einschliesslich der Hilfe über das Welternährungsprogramm).

\subsection{Wichtigste humanitäre Aktionen 2005 und 2006}

\subsubsection{Wiederaufbau nach dem Tsunami (ab Januar 2005)}

Nach der Tsunamikatastrophe vom Dezember 2004, mit Seebeben und Flutwelle in rund zehn asiatischen Ländern, haben die Einsätze der humanitären Hilfe ein Rekordniveau erreicht. Als Folge der Katastrophe wurden Höchstbeträge an privaten Mitteln durch Sammlungen in der Öffentlichkeit zusammengetragen. Ende Juni 2005 erreichten die Spendeneingänge der Glückskette allein 228 Millionen Franken ${ }^{9}$, ohne die direkten Spendenzahlungen an verschiedene Hilfswerke zu berücksichtigen ${ }^{10}$. Ende November 2006, fast zwei Jahre nach dem Tsunami, hat die Glückskette in Bezug auf die dank der Spenden finanzierten Projekte Bilanz gezogen. Demgemäss wurden Projekte im Gesamtbetrag von 187,9 Millionen Franken vor Ort durchgeführt, und 40 Millionen Franken sind für bereits festgelegte Projekte vorbehalten. Somit habe es laut Glückskette - im Gegensatz zu den kurz nach der Spendensammlung geäusserten Befürchtungen - nicht zuviele Spenden gegeben. Die von der Glückskette finanzierten und von den Hilfswerken vor Ort durchgeführten Projekte konzentrierten sich auf Sri Lanka (42 Projekte in Höhe von 77,3 Millionen Franken), Indonesien 
(29 Projekte im Umfang von 66,7 Millionen Franken), Indien (38,3 Millionen Franken), Thailand (3,7 Millionen Franken), Somalia (1,8 Millionen Franken) sowie auf die Hilfe für von der Katastophe betroffene Schweizer.

Die Humanitäre Hilfe des Bundes für die Nothilfeaktionen in sieben von der Katastrophe betroffenen Ländern belief sich auf insgesamt 28 Millionen Franken, davon 25 Millionen Franken in Form eines vom Bundesrat bereitgestellten Nachtragskredits. Infolge der Zerstörung von Strassen, Brücken und Häfen gab es bei der Beförderung der Nothilfe grosse Schwierigkeiten. Ein Dutzend Staaten, darunter die Schweiz, stellten insgesamt rund hundert Helikopter für den Transport der internationalen Hilfe vor Ort zur Verfügung. In der Rehabilitationsphase konzentrierte sich die Humanitäre Hilfe des Bundes auf den Wiederaufbau von Schulen und Wohnhäusern (Sri Lanka, Thailand), auf den Bau von Fischerbooten (Thailand) und auf die Instandsetzung der Abwasseraufbereitungsanlage in Bandah Aceh (Indonesien). In Sri Lanka unterstützte ein Konsortium von Schweizer Institutionen das Programm der Regierung zum Wiederaufbau von Wohnhäusern (Cash for Rehabilitation Programme). Dank dieses innovativen Hilfssystems konnten den von der Zerstörung ihrer Wohnungen Betroffenen direkte Geldbeträge ausgezahlt werden. Die Realisierung dieses Programms in zwei Distrikten wurde von der DEZA, der Glückskette, dem Schweizerischen Roten Kreuz (SRK) und dem Hilfswerk der Evangelischen Kirchen der Schweiz (HEKS) gemeinsam betreut. Die DEZA brachte drei Millionen Franken und die Glückskette 15 Millionen Franken auf, um den Wiederaufbau bzw. die Instandsetzung von rund 8500 Wohnhäusern bis Ende 2006 zu ermöglichen. Diese Hilfe wird noch bis mindestens Ende 2007 fortgesetzt.

Zwei Fischerdörfer auf den thailändischen Inseln Ko Phra Thong und Ko Kho Khao wurden am 2. Dezember 2006 eingeweiht. Der Entscheid betreffend diese Hilfe war im Anschluss an den Besuch von Micheline Calmy-Rey in der Region nach dem Tsunami getroffen worden. Die Fischerdörfer wurde völlig wieder instand gesetzt dank der Schweizer Hilfe, die dieses Projekt mit 4 Millionen Franken finanzierte (2 Millionen Franken durch die Glückskette, 1,7 Millionen Franken durch die DEZA und 300000 Franken durch private Spenden).

Global haben zahlreiche Journalisten und mehrere Studien die Probleme hervorgehoben, die mit dem besonders massiven Zufluss an Finanzmitteln nach der Tsunamikatastrophe verbunden waren: Anhäufung des entsandten Materials auf den Flughäfen, schwierige Koordination der Hilfe mit der Präsenz zahlreicher staatlicher, internationaler und nichtstaatlicher Akteure vor Ort, scharfe Konkurrenz unter NRO, Teil der Hilfe unangepasst für die wirklichen Bedürfnisse vor $\mathrm{Ort}^{11}$. Insgesamt wurden mehr als 13,5 Milliarden Dollar durch die internationale Gemeinschaft nach dieser Katastrophe zusammengetragen.

[D] Jahrbuch 2006, Nr. 1, Kapitel 4, 4.3.1. Humanitäre Aktion nach dem Tsunami, S. 54-57.

\subsubsection{Humanitäre Krise in Darfur (Sudan)}

Die Anzahl der Toten seit Beginn des Krieges im Darfurgebiet im Jahr 2003 wird auf 200000 geschätzt. 240000 Menschen leben in Flüchtlingslagern im

11 Vgl. beispielsweise die Untersuchung von Richard Werly, Tsunami. La vérité humanitaire, Paris, Editions du Jubilé, 2005. Siehe auch: „Tsunami : réquisitoire contre les ratés de l'aide internationale“, $L e$ Temps, 15. Juli 2006. 
Tschad und insgesamt zwei Millionen Menschen (d.h. ein Drittel der Bevölkerung Darfurs) ergriffen die Flucht. Eine halbe Million Menschen sind von der internationalen Hilfe abgeschnitten ${ }^{12}$. Die Situation für die Zivilbevölkerung ist weiterhin sehr prekär, trotz des 2005 abgeschlossenen Friedensabkommens, das allerdings nur von einem Teil der betroffenen Parteien im Jahr 2006 unterzeichnet wurde. Der Schutz und die Versorgung gestalten sich wegen der andauernden Kampfhandlungen schwierig, und die Helfer sind infolge der wiederholten Verletzungen des humanitären Völkerrechts grossen Gefahren ausgesetzt ${ }^{13}$. Seit 2004 hat die Schweiz 22 Millionen Franken an Hilfeleistungen für Flüchtlinge oder Vertriebene aufgebracht, namentlich durch die Finanzierung von Projekten des Welternährungsprogramms (WFP), des IKRK, des UN-Hochkommissariats für Flüchtlinge (UNHCR), der Weltgesundheitsorganisation (WHO) und der NRO Medair.

\subsubsection{Erdbeben in Pakistan und im Kaschmirgebiet (Oktober 2005)}

Anfang Oktober 2005 hatte ein heftiges Erdbeben das Kaschmirgebiet in Teilen Pakistans und Nordindiens erschüttert. Dabei handelte es sich um eines der stärksten Beben in der Geschichte Pakistans mit über 87000 Todesopfern, 77000 Schwerverletzten und rund drei Millionen Obdachlosen bei Herannahen des Winters. Die Glückskette finanzierte in einer ersten Phase ein Dutzend Projekte, die in Pakistan durch sechs Hilfswerke verwaltet wurden (Verteilung von Nothilfematerial und Notunterkünften, Trinkwasserversorgungssystemen, Holzheizungen). Die darauf folgende Wiederaufbauphase umfasste die Wiedererrichtung von Wohnhäusern, Schulen und Gesundheitszentren sowie psychosoziale Unterstützung für Kinder und Jugendliche. Drei Viertel der Spendensammlung der Glückskette, welche zwölf Millionen Franken erreichte, werden für die Nothilfe und ein Viertel für die Wiederaufbauphase eingesetzt.

Von der DEZA wurden elf Millionen Franken für Nothilfe, davon fünf Millionen über UN-Organisationen aufgebracht. In dieser Phase wurde das Schweizerische Korps für humanitäre Hilfe (SKH) für ärztliche Nothilfe und zur Verteilung von Zelten, Decken, Medikamenten und Kochgeräten vom Bund entsandt. In einer zweiten Phase wurden provisorische Bauten für Schulen und Gesundheitsposten errrichtet. Auch wurde Baumaterial und Werkzeug für den Bau temporärer Unterkünfte zur Verfügung gestellt.

Anschliessend engagierte sich die DEZA mit 22 Millionen Franken in der von 2006 bis 2009 vorgesehenen Wiederaufbauphase, insbesondere für den Wiederaufbau von Infrastruktureinrichtungen in rund fünfzig Dörfern und für die Ausbildung von technischem Personal für erdbebenfeste Bauten ${ }^{14}$.

12 Zahlen aus einem Artikel der DEZA, Krise in Darfur. Die Friedensappelle verhallen ungehört, 8. November 2006.

13 Ibid. Siehe auch das Gespräch mit Hansjürg Ambühl, HH+SKH - Sektion Afrika der DEZA, Krise in Darfur. Die Friedensappelle verhallen ungehört; ferner den Artikel „Kein Ende der Gesetzlosigkeit in Darfour“, Neue Zürcher Zeitung, 15.-16. April 2006.

14 Quellen: DEZA, „Erdbeben im Kaschmir. Der Wiederaufbau verläuft planmässig“, Medienmitteilung, 19. April 2006. Siehe auch auf der Website der DEZA unter <http://www.deza.admin.ch>, Merkblatt zum Erdbeben in Pakistan vom 14. Dezember 2005 ( $>$ Länder $>$ Pakistan $>$ Erdbeben in Kaschmir sowie die gesamte Hilfe der Schweiz in Pakistan (unter $>$ Länder $>$ Pakistan), bzw. die Internetseite DEZA-Pakistan: <http://www.sdcpakistan.org>. 


\subsubsection{Erdbeben auf Java (Mai 2006)}

Das Erdbeben auf Java hat mehr als 6000 Tote, 15000 Verletzte und über 150000 Obdachlose gefordert. Ende Mai 2006 gaben die Schweizer Hilfswerke eine Million Franken zugunsten der Opfer für Nothilfe und Wiederaufbau frei. Die DEZA engagierte sich mit 250000 Franken, und 130000 Franken wurden an das indonesische Rote Kreuz ausgezahlt.

\subsubsection{Bewaffneter Konflikt im Libanon (Sommer 2006)}

Nach dem bewaffneten Konflikt im Libanon im Juli 2006 und bis Ende September 2006 hat die Schweiz Hilfe für die Opfer finanziert, um die Rückkehr der internen Flüchtlinge in ihre Dörfer zu fördern, Schulen wieder instand zu setzen $^{15}$, medizinische Versorgung zu leisten und nach der Verseuchung der Küstengewässer infolge der Bombardierung eines Treibstoffdepots Unterstützung zu gewähren ${ }^{16}$.

Die Schweiz beteiligt sich an den Entminungsaktionen im Libanon mit Systemen zur Entschärfung von Minen und nicht explodierten Bomben (Blindgängern). Das Eidgenössische Departement für Verteidigung, Bevölkerungsschutz und Sport (VBS) und die DEZA unterstützen die libanesische Regierung durch die Entsendung von Minenräummaterial (Spezialsysteme SM EOD), das die Beseitigung von Minen und Blindgängern ohne direkte Handhabung gestattet. Daneben werden Schutzkleidung und Metalldetektoren geliefert. Ferner beteiligt sich die Schweiz an der Ausbildung von Entschärfungsfachleuten. Gemäss den Vereinten Nationen sind die Felder und Olivenhaine im Südlibanon mit über einer Million nicht detonierter Streubomben (zwei pro Einwohner) verseucht. Dabei handelt es sich um schwer aufzufindende Sprengkörper, die eine umfangreiche Entminungsarbeit erfordern. (Im Kosovo beispielsweise wurden 25000 Bomben in über zwei Jahren entschärft.) Insgesamt wurden 16 Millionen Dollar für humanitäre Hilfe im Nahen Osten eingesetzt. Die Hilfe für Palästina wird zum Grossteil über das Programm der Vereinten Nationen für die Palästinaflüchtlinge (UNWRA) geleitet.

\subsection{Tätigkeit verschiedener Akteure der humanitären Hilfe}

\subsubsection{Jahrestagung der Humanitären Hilfe 2006}

Diese öffentliche Jahresveranstaltung bringt die Partner der Humanitären Hilfe der DEZA zusammen. Die Jahrestagung der Humanitären Hilfe 2006 fand am 7. April 2006 statt und war dem Thema der Vertriebenen infolge von Konflikten und Katastrophen gewidmet. Am Beispiel von vier Ländern wurden die Auswirkungen von Zwangswanderungen auf die betroffenen Bevölkerungsgruppen veranschaulicht. Bezüglich Uganda wurde auf die Bedeutung des Schutzes der Kin-

15 Instandsetzung von Schulen, die durch Bombardierungen beschädigt wurden oder zur Aufnahme der Vertriebenen eingesetzt wurden.

16 DEZA, „Humanitäre Hilfe der Schweiz im Libanon“, Pressemitteilung vom 29. September 2006 sowie das der Mitteilung beigefügte Merkblatt, <http://www.deza.admin.ch $>>$ Aktuell $>$ News $>$ Suche nach Stichwort „Libanon“. 
der gegen die militärische Zwangsrekrutierung hingewiesen. In Bosnien-Herzegowia stellt sich das Problem der schwierigen Integration ethnischer Minderheiten. Der Vertreter der UNWRA behandelte das Thema der Hilfe für die Palästinaflüchtlinge. Ferner wurde das Engagement der Schweiz für die Flüchtlinge aus Myanmar in Thailand angeschnitten.

\subsubsection{Sechzigjähriges Bestehen der Glückskette}

Die erste Radiosendung der Glückskette wurde am 26. September 1946 vom Westschweizer Rundfunk ausgestrahlt. Die wöchentliche Radiosendung wurde ab 1954 durch Sendungen anlässlich wichtiger Ereignisse - Naturkatastrophen oder Kriege - zum Zweck von Spendenaufrufen ersetzt. Im Verlauf ihres sechzigjährigen Bestehens hat die Glückskette über 900 Millionen Franken im Rahmen von 115 Sammelaktionen zusammengetragen. Die Glückskette führt die Projekte vor Ort nicht selber durch, sondern prüft die Vorschläge und gewährt Mittel für Projekte, die von den 32 Schweizer Partnerorganisationen eingereicht werden. Im Durchschnitt werden 15 Prozent der Spenden für Nothilfe im engeren Sinne, 70 Prozent für Wiederaufbauprojekte und 15 Prozent für längerfristige Entwicklungsprojekte eingesetzt. 2005 hat die Glückskette die Rekordsumme von 272 Millionen Franken zusammengetragen, davon 204 Millionen Franken nach dem Tsunami in Südasien ${ }^{17}$, 49,2 Millionen Franken nach den Unwettern in der Schweiz (August 2005), 10,9 Millionen Franken nach dem Erdbeben in Kaschmir und 2,3 Millionen Franken nach dem Wirbelsturm Stan in Mittelamerika. 1,1 Millionen Franken kamen für den Sudan und 750000 Franken für die Bekämpfung der Hungersnot in Afrika zusammen ${ }^{18}$.

\subsubsection{Internationales Komitee vom Roten Kreuz (IKRK)}

Die 29. Internationale Konferenz des Roten Kreuzes und Roten Halbmonds, die im Juni 2006 stattfand, änderte die Statuten der Internationalen Rotkreuz- und Rothalbmondbewegung, um das zusätzliche Emblem des roten Kristalls aufnehmen zu können. Im Anschluss an diese Entscheidung hat das IKRK die nationale israelische Gesellschaft (Magen David Adom) und den palästinensischen Roten Halbmond anerkannt. Die diplomatische Konferenz der Staaten hatte im Dezember 2005 das dritte Zusatzprotokoll zu den Genfer Konventionen angenommen, das ein zusätzliches Emblem unter dem Namen ,roter Kristall“ einführte. Die Schweiz hatte als Depositarstaat der Genfer Konventionen eine wichtige Rolle bei der Vorbereitung der Annahme des Protokolls gespielt ${ }^{19}$.

[Dahrbuch 2006, Nr. 1, Kapitel 4, 4.5. Neues Emblem für die Internationale Rotkreuz- und Rothalbmondbewegung, S. 61-62.

Die Schweiz gehört zu den Hauptgeldgebern des IKRK. 2005 waren die wichtigsten Geber die Vereinigten Staaten (200 Millionen Franken), Grossbritannien (100 Millionen Franken), die Schweiz (93 Millionen Franken), die Europäische

17 23,4 Millionen Franken für die Tsunamiopfer waren in den letzten Tagen des Jahres 2004 eingegangen.

18 Glückskette, Jahresbericht 2005, 2006, <http://www.glueckskette.ch>.

19 „Le ,Cristal rouge“ doit encore passer son baptême du feu“, Le Temps, 20. Juni 2006. „La Croix-Rouge toujours divisée sur son emblème“, Le Temps, 22. Juni 2006. „La Croix-Rouge accepte le cristal et accueille Israéliens et Palestiniens“, Le Temps, 23. Juni 2006. 
Kommission (90 Millionen Franken), die Niederlande (60 Millionen Franken) und Schweden (50 Millionen Franken) ${ }^{20}$. Die Aufwendungen des IKRK für Aktionen im Feld haben im Jahr 2006 eine Milliarde Franken überschritten. Die wichtigsten Einsätze für 2006 betrafen den Libanon (101 Millionen Franken), Pakistan (97 Millionen Franken), Israel (einschliesslich der besetzten Gebiete, 53 Millionen Franken), Somalia (42 Millionen Franken), Afghanistan (39 Millionen Franken) und den Irak (38 Millionen Franken). In seinem Finanzierungsappell vom Dezember 2006 strebte das IKRK über eine Milliarde Franken an, um seine Tätigkeit im Jahr 2007 zu finanzieren (843 Millionen Franken für die Aktionen im Feld et 160 Millionen Franken zur Finanzierung seines Genfer Sitzes (800 Beschäftigte).

Im Dezember 2006 lehnte der Bundesrat einen Antrag auf Erhöhung des Bundesbeitrags an das IKRK ab ${ }^{21}$. Bundesrätin Micheline Calmy-Rey hatte vorgeschlagen, den Jahresbeitrag an das IKRK um 28 Millionen Franken aufzustocken, womit sich der Beitrag ab 2008 auf 120 Millionen Franken belaufen hätte. Dies, um den steigenden Bedürfnissen Rechnung zu tragen und die Position der Schweiz als zweitgrösster Geldgeber des IKRK nach den USA zu festigen.

\section{QUELLEN}

Bundesrat, Botschaft über die Weiterführung der internationalen humanitären Hilfe der Eidgenossenschaft, vom 29. November 2006, (BB1 2006, 9617).

Caritas, Les défis humanitaires. Les dilemmes politiques de l'aide d'urgence, coll. Prise de position, $\mathrm{Nr}$. 11, Luxemburg, Caritas Luxemburg; Luzern, Caritas Schweiz, November 2005, 164 S.

DEZA, Pressemitteilungen 2006.

DEZA/SECO, Jahresbericht der internationalen Zusammenarbeit der Schweiz, 2005, Bern, 2006, 24 S.

Glückskette, Jahresbericht 2005, 2006.

\section{INTERNET-ADRESSEN}

Direktion für Entwicklung und Zusammenarbeit (DEZA) : <www.deza.admin.ch > (>Humanitäre Hilfe).

Glückskette: <www.glueckskette.ch>.

Internationales Komitee vom Roten Kreuz (IKRK) : <www.icrc.org $>$.

Médecins sans frontières: <http://www.msf.ch>.

UN-Büro für die Koordination humanitärer Angelegenheiten (OCHA) : <http://ochaonline.un.org $>$.

Welternährungsprogramm (WFP): <http://www.wfp.org >.

20 Quelle für diesen Abschnitt: CICR, Vue d'ensemble des opérations du CICR 2007, <http://www.icrc. org/fre/appels-2007> (Stand Dezember 2006).

21 „Le Conseil fédéral chipote sur son soutien annuel au CICR“, Le Temps, 4. Dezember 2006. 